\title{
Effects of boron doping on the surface morphology and structural imperfections of diamond films
}

\author{
X. H. Wang, G.-H. M. Ma, Wei Zhu and J. T. Glass \\ Department of Materials Science and Engineering, North Carolina State University, Raleigh, NC 27695-7919 (USA) \\ L. Bergman, K. F. Turner and R. J. Nemanich \\ Department of Physics, North Carolina State University, Raleigh, NC 27695-8202 (USA)
}

(Received October 11, 1991; in final form January 3, 1992)

\begin{abstract}
This paper reports the surface morphology and structural imperfection of boron-doped diamond films prepared by microwave plasma enhanced chemical vapor deposition. It was found that boron dopants improved the structural quality of diamond films. The surface morphology consisted mainly of the $\{111\}$ facets. A significant enhancement of nucleation density and consequent decrease of grain size was observed with the addition of diborane in the gas phase. Raman spectroscopy indicated that, with the introduction of boron dopants, the integrated intensity of the diamond peak at $1332 \mathrm{~cm}^{-1}$ increased relative to the intensity of the non-diamond peak at about $1500 \mathrm{~cm}^{-1}$, and the full-width at half maximum of the $1332 \mathrm{~cm}^{-1}$ peak decreased. In addition, the $1.681 \mathrm{eV}(738 \mathrm{~nm})$ photoluminescence peak related to point defects was effectively reduced, or even eliminated by the boron dopants. Finally, transmission electron microscopy studies found that the densities of planar defects (mainly stacking faults and microtwins) also decreased with the boron addition.
\end{abstract}

\section{Introduction}

There are extensive research activities in the growth of $p$-type semiconducting diamond films by boron doping in chemical vapor deposition (CVD) processes [1-6]. The electrical characteristics of boron-doped diamond films have been widely reported. However, little is known about the effects of boron doping on the microstructure of such p-type diamond films. Various microstructural features can be either directly responsible for or related to the desired film properties. It has been shown that vapor deposited diamond films contain extensive crystalline defects (stacking faults, microtwins and dislocations) and other structural imperfections (secondary phases, grain boundaries, surface roughness, impurities, etc.) [7-11]. These structural defects degrade many physical properties of CVD diamond including optical transparency, electrical resistivity and thermal conductivity $[12,13]$. A comprehensive characterization and understanding of the microstructure of CVD diamond films and its correlations with the process parameters (in this study, the boron dopant) is thus of great importance in an attempt to eventually control and tailor the microstructure of diamond films for specific applications. This will, in turn, lead to improved electrical properties of such boron-doped, $p$-type diamond films and enhance their applications in the semiconductor industry.

In 1988, Kobashi et al. [3] observed that the film morphology was sensitive to the diborane concentration in the $\mathrm{CH}_{4} / \mathrm{H}_{2}$ gas phase. With the increase of the diborane concentrations, the surface morphology changed from the randomly oriented, triangular $\{111\}$ facets to the pyramidal $\{111\}$ facets and then to a needle-like graphitic structure. Fujimori et al. $[1,5]$ reported that the crystallinity of homoepitaxial diamond films was affected by boron doping. With electron energy loss spectroscopy (EELS) they showed that boron dopants $\left(10^{20}\right.$ atom $\left.\mathrm{cm}^{-3}\right)$ effectively eliminated the characteristic peak of graphite in the boron-doped diamond film. Nishimura et al. [6] and Yacobi et al. [14] also reported that the structural perfection of boron-doped diamond films was higher than undoped films. In this study, we report in detail on the effects of boron doping on the microstructure of diamond films. Electron microscopies, Raman spectroscopy and photoluminescence spectroscopy were used to reveal the various aspects of the microstructural features of diamond films. The results were related to the reported electrical measurements of boron-doped diamond films. Possible mechanisms responsible for the boron effects on the microstructure of diamond films were discussed. 


\section{Experimental details}

Boron dopants were introduced into diamond films by mixing diborane $\left(\mathrm{B}_{2} \mathrm{H}_{6}\right)$ with methane $\left(\mathrm{CH}_{4}\right)$ and hydrogen $\left(\mathrm{H}_{2}\right)$ gases in a microwave plasma-enhanced CVD process. The details of the deposition apparatus and experimental procedures have been given previously [15]. A series of films were prepared with different doping levels by varying the diborane concentration in the gas phase from 0 to $1 \mathrm{ppm}(0,0.005,0.01,0.1,0.5$ and $1 \mathrm{ppm}$; corresponding $\mathrm{B} / \mathrm{C}$ ratios were $0,2,4,40$, 200 and $400 \mathrm{ppm}$ respectively). The substrates were $p$ type $\langle 111\rangle$ oriented single-crystalline silicon wafers (resistivity of less than $1 \Omega \mathrm{cm}$ ). They were pretreated by polishing with $0.25 \mu \mathrm{m}$ diamond paste for $1 \mathrm{~h}$ followed by ultrasonic cleaning in distilled water, alcohol and acetone. The major process parameters for the growth of these films were: substrate temperature, $800^{\circ} \mathrm{C}$; pressure, 35 Torr; methane-to-hydrogen volumetric ratio, $0.5 \%$; total gas flow rate, $100 \mathrm{~cm}^{3} \mathrm{~min}^{-1}$; deposition time, $7 \mathrm{~h}$. The thicknesses of these films were approximately $1.1 \mu \mathrm{m}$, corresponding to an average growth rate of about $0.15 \mu \mathrm{m} \mathrm{h}^{-1}$.

Secondary ion mass spectrometry (SIMS) was used to quantitatively determine the boron concentration in the films, with a boron ion-implanted type IIa diamond single crystal as standard (with a dose of $10^{15} \mathrm{atom} \mathrm{cm}^{-3}$ implanted at $150 \mathrm{keV}$ ). Scanning electron microscopy (SEM) and scanning tunneling microscopy (STM) were used to examine the surface morphology of the films. Transmission electron microscopy (TEM) with parallel detection electron energy-loss spectroscopy (EELS) was employed to investigate the internal structural imperfections and the segregation of boron dopants in the films. Finally, Raman spectroscopy and photoluminescence spectroscopy were used to study the diamond crystallinity and optically active point defects within the films. The spectra from both the Raman region (1000$\left.1800 \mathrm{~cm}^{-1}\right)$ and the photoluminescence region $(1000$ $7000 \mathrm{~cm}^{-1}$ ) of diamond were excited using a $514.53 \mathrm{~nm}$ argon ion laser light. The laser beam had a spot size of about $2 \mu \mathrm{m}$ which sampled about $2-5$ grains in the current diamond films.

\section{Results}

\subsection{Boron concentrations in the diamond films}

Three diamond films deposited with $\mathrm{B} / \mathrm{C}$ ratios of 0 , 40 and $400 \mathrm{ppm}$ in the $\mathrm{CH}_{4} / \mathrm{H}_{2}$ gas phase were measured by SIMS. The SIMS measurements were performed with a $15 \mathrm{keV} \mathrm{O}_{2}$ beam. The sputtered area was $250 \times 250 \mu \mathrm{m}^{2}$ and the sampling diameter was about $60 \mu \mathrm{m}$. The measured boron concentrations in these three films were 0 , 0.49 and $33.6 \times 10^{18}$ atom $\mathrm{cm}^{-3}$ respectively. These values correspond to $\mathrm{B} / \mathrm{C}$ ratios in the diamond films of 0,3 and $190 \mathrm{ppm}$ respectively. The data suggest that the boron atoms effectively incorporated into the diamond films increased with the $\mathrm{B}_{2} \mathrm{H}_{6}$ concentration in the gas phase. The chemical mapping image of boron atoms in the film grown with a $\mathrm{B} / \mathrm{C}$ ratio equal to $400 \mathrm{ppm}$ showed that boron atoms were dispersed uniformly throughout the diamond film. However, considering the spatial resolution of the SIMS chemical mapping technique, which was about $1 \mu \mathrm{m}$ and larger than the grain sizes of the current diamond films, possible aggregations of boron atoms near the grain boundaries might simply not be resolved. Consequently, EELS in TEM was used to detect the boron distribution in the diamond grains and to check if boron segregation along grain boundaries occurred. No boron was detected in all the EELS spectra collected either inside the diamond grains or along the grain boundaries. Thus, it is believed that any boron segregation which may be present in the current diamond films was below the minimum detectable concentration of boron in a carbon matrix by EELS which is about 0.2 at. \% over the probed region (about $50 \AA$ diameter spot size) [16]. In addition, electron diffraction patterns taken in TEM also revealed no evidence of the formation of any boron carbide. This is not surprising since the incorporated boron concentration was apparently too low to form a second phase.

It should be noted that the boron concentrations in the films were lower than the boron concentrations in the gas phase, indicating that the incorporation rate of boron species was smaller than that of the carbon species. This is opposite to what was reported for boron doping of diamond films grown on alumina $\left(\mathrm{Al}_{2} \mathrm{O}_{3}\right)$ substrates [6] in which the boron concentrations in the films were greater than the boron concentrations in the precursor gases. This can possibly be attributed to the use of different substrate materials (silicon vs. $\mathrm{Al}_{2} \mathrm{O}_{3}$ ) which have differing effects on the nucleation and growth rates of diamond. Other minor impurities found in the films included hydrogen, nitrogen and silicon. They were present in both doped and undoped films, and their concentrations did not change with the addition of boron dopants except for the hydrogen concentrations which slightly decreased. The accurate values of the concentrations of these impurities were not available because of the lack of standard samples.

\subsection{Surface morphology}

The surface morphology of undoped diamond films consisted mainly of the triangular $\{111\}$ facets plus a few $\{100\}$ facets as shown in Fig. 1(a). As boron concentration increased, the number of $\{100\}$ facets dispersed in this $\{111\}$ morphology decreased. Thus, it appears that boron atoms promote or stabilize the $\{111\}$ faces as the dominant surface morphology (compare Fig. 1(a) with Figs. 


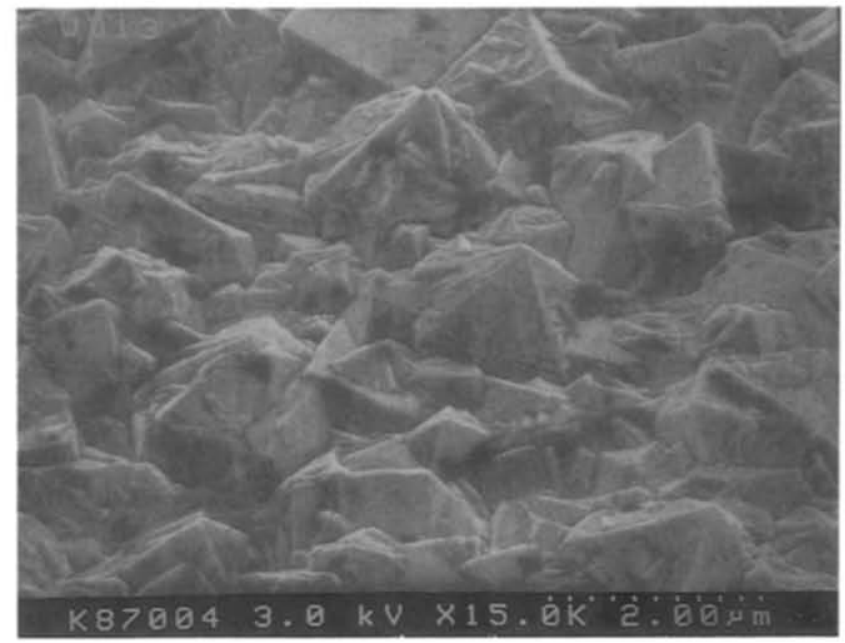

(a)

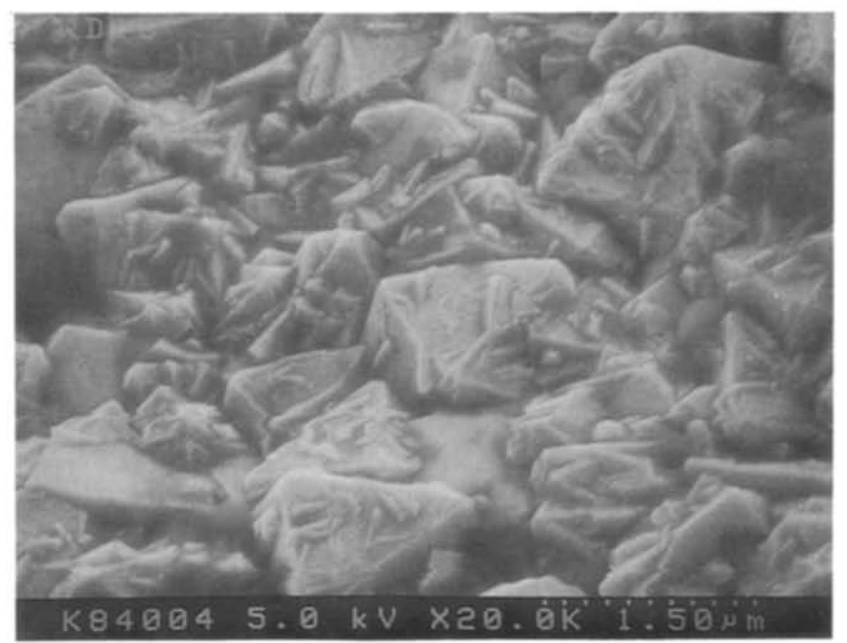

(b)

Fig. 1.

1(e) and 1(f)). However, since the low methane concentration employed in the deposition process $(0.5 \%)$ also tends to favor the formation of the $\{111\}$ faces on the surface [15], further experiments under higher methane concentration (e.g. $2.0 \%$ ) conditions are necessary to verify this. It will also be helpful to determine if the concentration and distribution of boron atoms are different between the $\{111\}$ faces and the $\{100\}$ faces. Such results would be useful in establishing the role of boron in controlling the crystallographic development of diamond.

The grains of heavily doped films (Figs. 1(e) and 1(f)) had a more regular shape and smoother surface than the undoped or slightly doped films which exhibited various growth features on the surface (see Figs. 1(a) and $1(b)$ ). This is believed to be related to an enhanced surface mobility of carbon species with the introduction of boron as discussed in section 4.1. The boron dopants

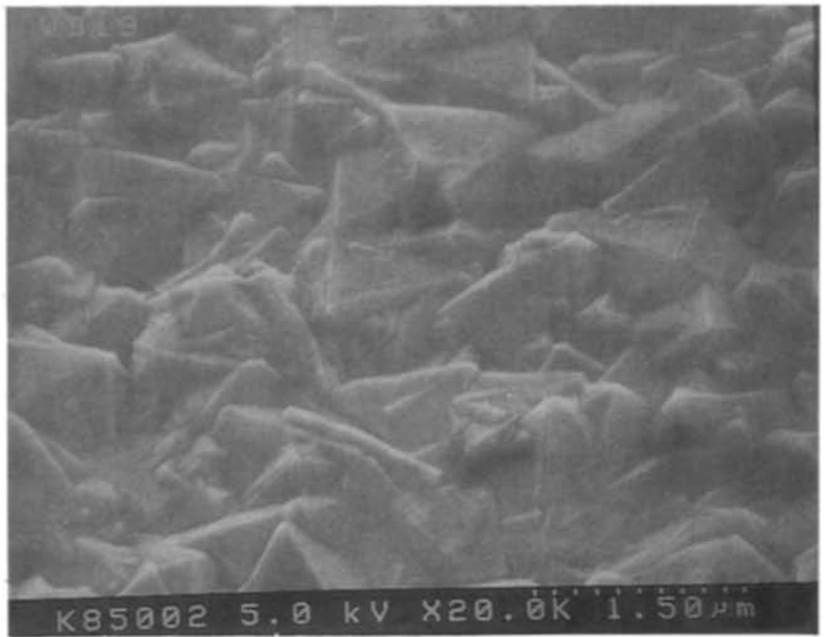

(c)

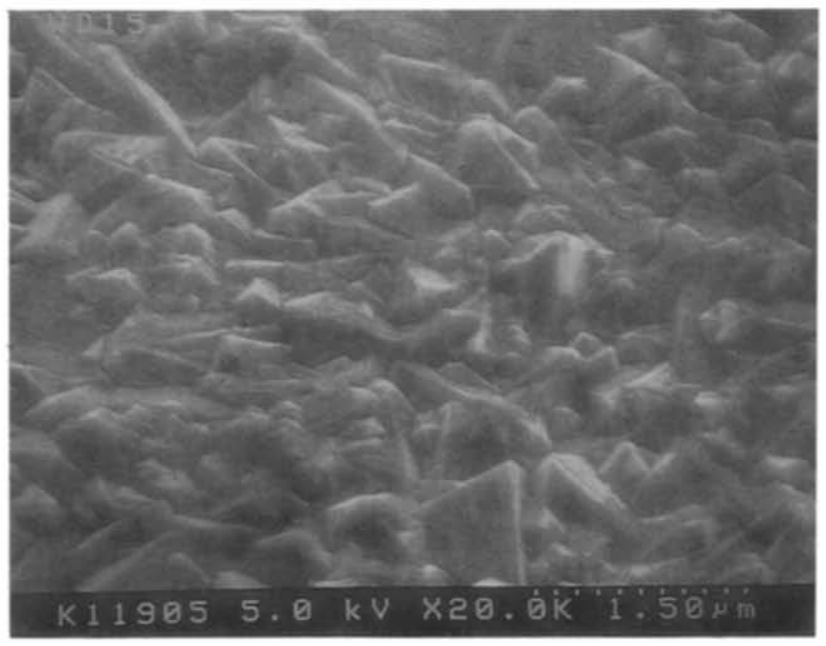

(d)

also greatly enhanced the nucleation density of diamond films as shown in Fig. 1, in which a significant decrease of grain sizes can be observed with the increase of the $\mathrm{B}_{2} \mathrm{H}_{6}$ concentrations in the gas phase. The grain sizes changed from about $1 \mu \mathrm{m}$ for undoped or lightly doped (B/C ratios at 2-40 ppm) films (Figs. 1(a), 1(b) and 1(c)) to about $0.5 \mu \mathrm{m}$ for heavily doped films (greater than $40 \mathrm{ppm}$ ) (Figs. 1(d), 1(e) and 1(f)).

Scanning tunneling microscopy (STM) was successfully performed on the diamond films grown with ratio $\mathrm{B} / \mathrm{C}>4 \mathrm{ppm}$, and it provided information on the surface topography on a smaller scale than the SEM results. The tunneling current and the biasing voltage used for imaging were about $1.0 \mathrm{nA}$ and -1.0 to $-2.1 \mathrm{~V}$ respectively. The STM topographic data correlated with the corresponding SEM results. Figure 2 exhibits STM topographs of the surfaces of diamond films grown at the $\mathrm{B} / \mathrm{C}$ ratios of 4 and $400 \mathrm{ppm}$. The average surface 


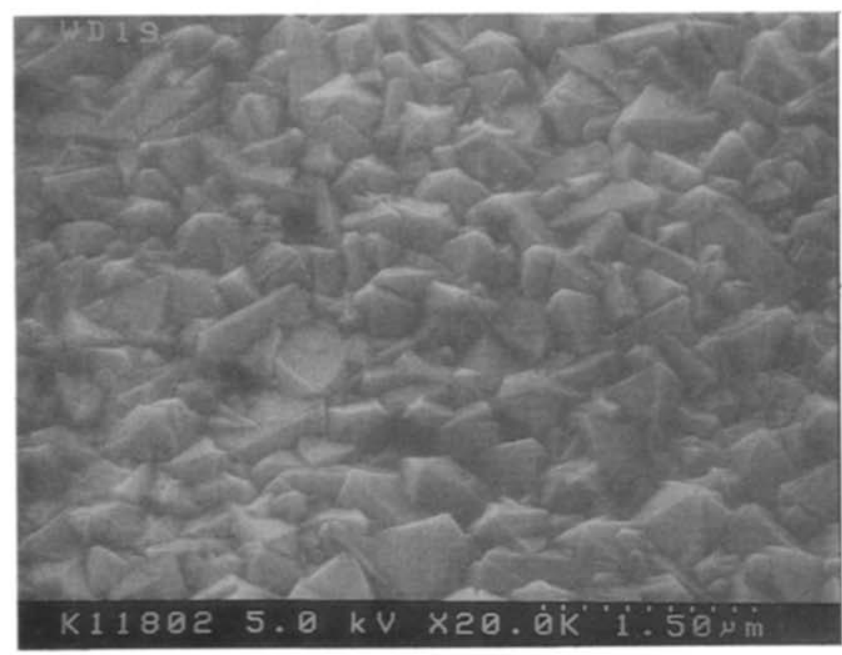

(e)

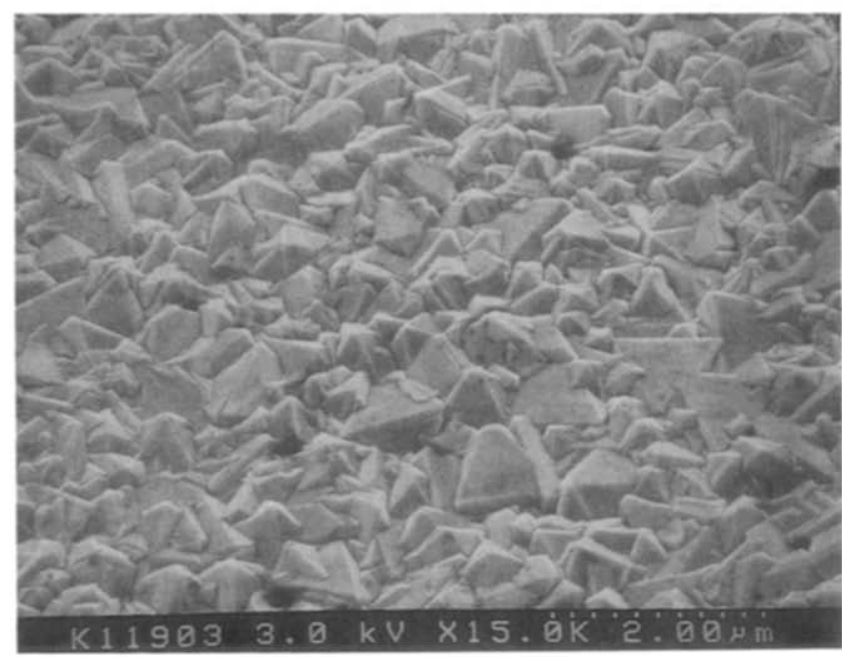

(f)

Fig. 1. SEM images of the growth surfaces of diamond films grown at different B/C ratios: (a) 0 ppm. (b) $2 \mathrm{ppm}$, (c) 4 ppm. (d) $40 \mathrm{ppm}$. (e) $200 \mathrm{ppm}$, (f) $400 \mathrm{ppm}$. It appears that boron atoms promote or stabilize the $\{111$ faces as the dominant surface morphology. A significant decrease of grain sizes can be observed with the increase of the $\mathrm{B}_{2} \mathbf{H}_{6}$ concentration in the gas phase.

roughness (about $0.5 \mu \mathrm{m}$ for the ratio of $4 \mathrm{ppm}$ and about $0.3 \mu \mathrm{m}$ for the ratio of $400 \mathrm{ppm}$ ) was comparable with that observed from SEM. However, individual grains had much smoother surfaces than the overall film (about $28 \AA$ for the ratio of $4 \mathrm{ppm}$ and about $18 \AA$ for the ratio of $400 \mathrm{ppm}$ ). In addition, ridges and steps oriented parallel to each other were observed which represent features of twinning bands within the grains intersecting with the surface. The limitation of imaging by the STM technique for diamond films deposited at $\mathrm{B} / \mathrm{C}$ less than $4 \mathrm{ppm}$ was due to their poor electrical conductivities. These low electrical conductivities made it more difficult to define the spatial positions of carbon atoms on diamond surfaces because of the degraded

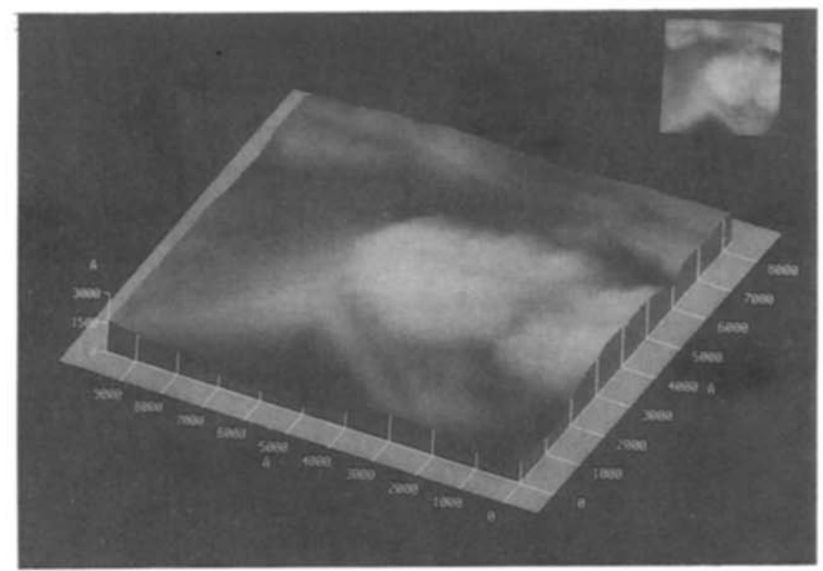

(a)

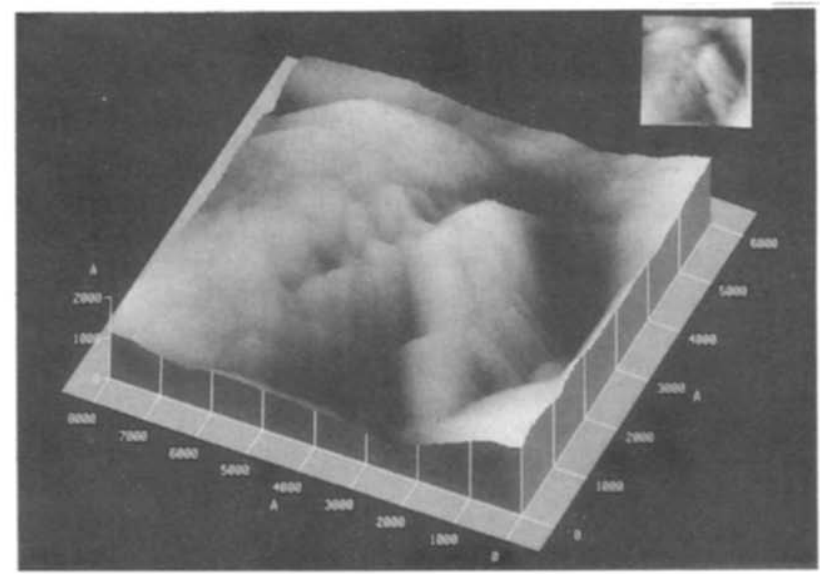

(b)

Fig. 2. STM topographs of the surfaces of the diamond films grown at $B$ C ratios of (a) $4 \mathrm{ppm}$, and (b) $400 \mathrm{ppm}$. The surface average roughness was comparable with that observed from SEM.

resolution resulting from both large fluctuations in the tunneling current during scans and frequent tip crashes into insulating regions [17. 18].

\subsection{Structural imperfections}

The Raman spectra shown in Fig. 3 indicated that, with the introduction of boron dopants, the integrated intensity of the diamond peak at $1332 \mathrm{~cm}^{-1}$ increased relative to the intensity of the non-diamond feature at $1500 \mathrm{~cm}^{-1}$, and the full-width at half maximum (FWHM) of the $1332 \mathrm{~cm}^{-1}$ peak decreased. It has been reported previously [19] that the microcrystalline domain sizes of diamond films can be calculated based on the phonon dispersion curves by utilizing the linewidth of the diamond peak at $1332 \mathrm{~cm}^{-1}$, although the existence of non-uniform stress may complicate the matter. The decreasing FWHM of the diamond peak reveals an increasing domain size of diamond, which indicates an improvement of the diamond structural perfection. In addition, the Raman spectral background 


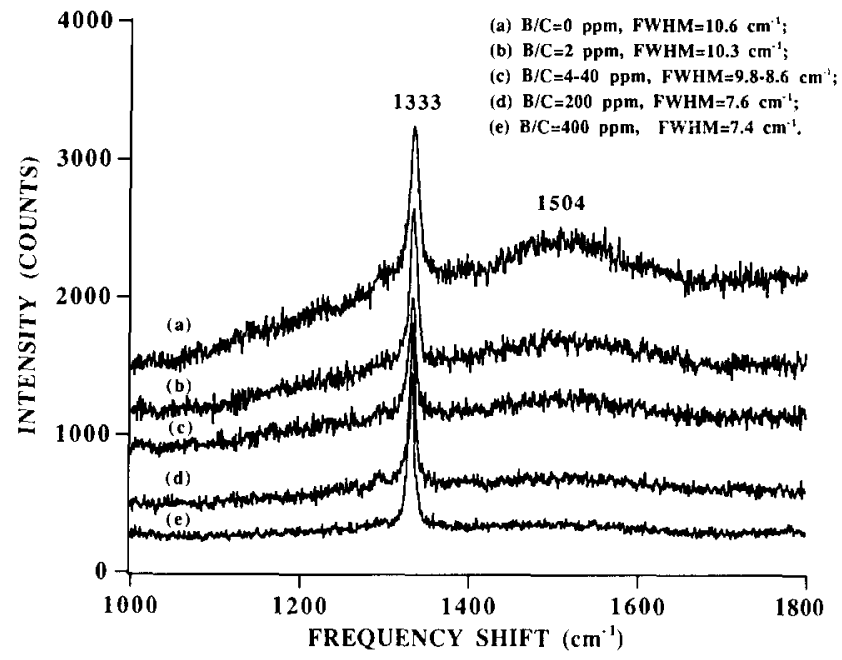

Fig. 3. Raman spectra of diamond films grown at different $\mathrm{B} / \mathrm{C}$ ratios. It shows that with the introduction of boron dopants, the diamond peak at $1332 \mathrm{~cm}^{-1}$ increased relative to the non-diamond feature at $1500 \mathrm{~cm}^{-1}$, and the FWHM of the $1332 \mathrm{~cm}^{-1}$ peak decreased.

arising from the luminescence also decreased with the boron addition. Therefore, the films doped with boron atoms contained a decreasing amount of non-diamond phases and possessed an increasing degree of structural perfection of diamond, despite the increased grain boundary area caused by the decreasing grain size. It is interesting to note that apparently this increased grain boundary area had no significant contributions to the spectral background. Therefore it can be surmised that lattice defects rather than grain boundaries are primarily responsible for the luminescent background.

The improvement of structural perfection by the boron addition has also been supported by the TEM results as shown in Fig. 4. It can be seen that the densities of planar defects (mainly stacking faults and microtwins) decreased when boron was doped into the films. Some diamond grains in the heavily doped films contained very few microtwins and stacking faults and were almost defect-free. The reduction of these planar defects is even more significant when one considers that the $\{111\}$ faceting increased with the boron addition, because the $\{111\}$ faces are well known to contain higher densities of planar defects than other crystallographic faces [20, 21]. Similar phenomena of defect reduction were observed in TEM sampling of other doped films with different dopant concentrations. Therefore it is believed that the defect density was globally reduced in the borondoped films. The results are rare in undoped diamond films grown under similar process conditions, especially in those $\{111\}$ faceted diamond grains which normally contain extensive planar defects $[7-11,20,21]$.

The effects of boron atoms on the luminescence features of CVD diamond films were particularly interesting. The photoluminescence (PL) spectra in Fig. 5

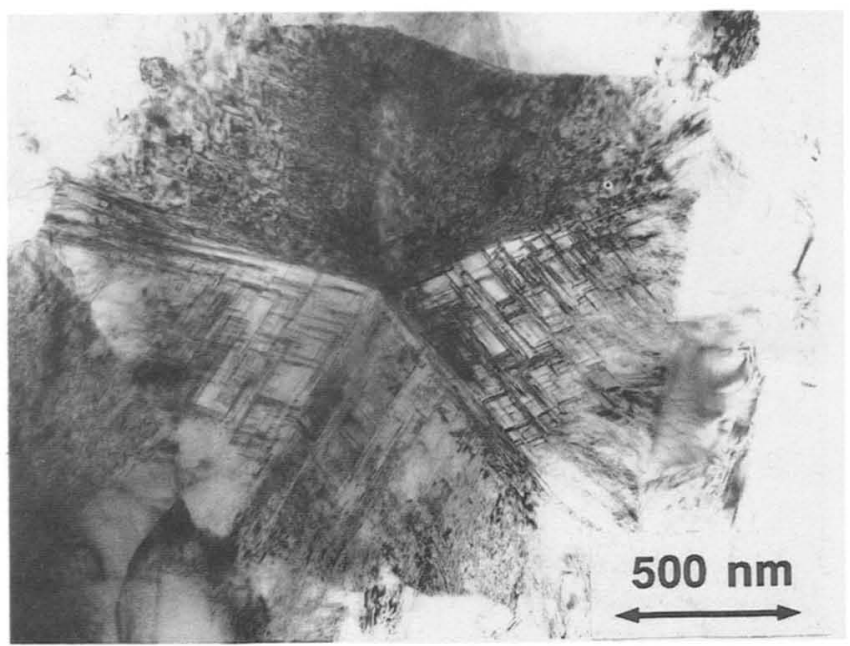

(a)

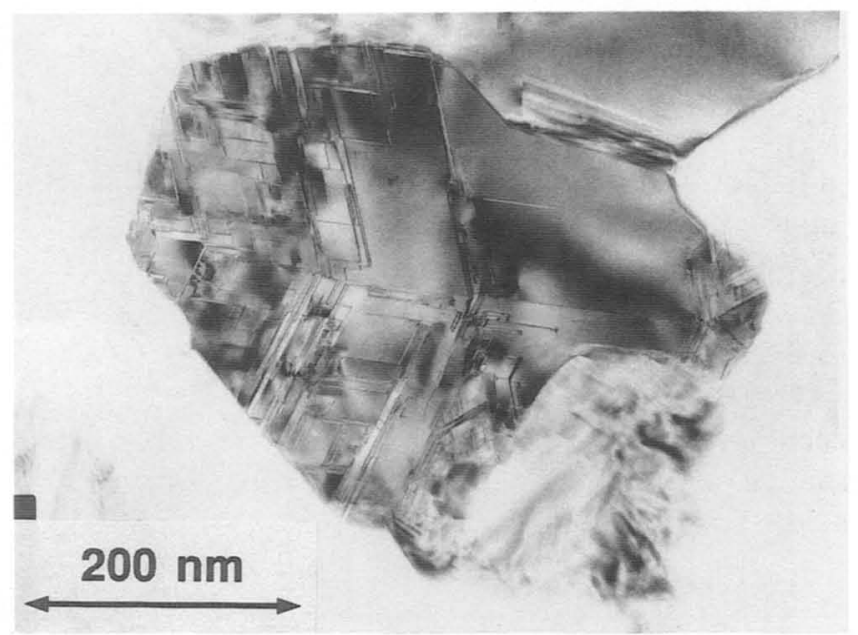

(b)

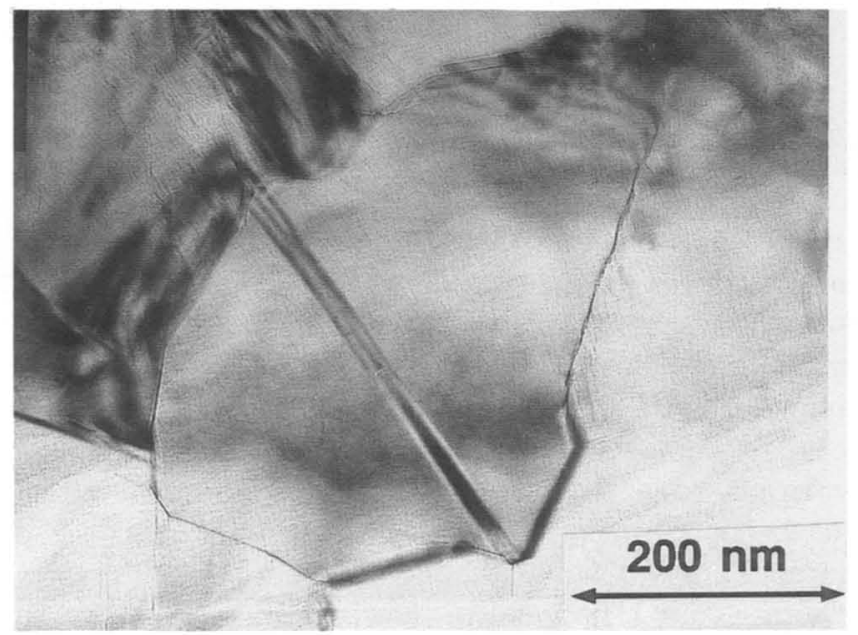

(c)

Fig. 4. TEM images of diamond films grown at different $\mathrm{B} / \mathrm{C}$ ratios: (a) $0 \mathrm{ppm}$, (b) $2 \mathrm{ppm}$, (c) $400 \mathrm{ppm}$. It can be seen that the densities of planar defects decreased with the introduction of boron dopants. 


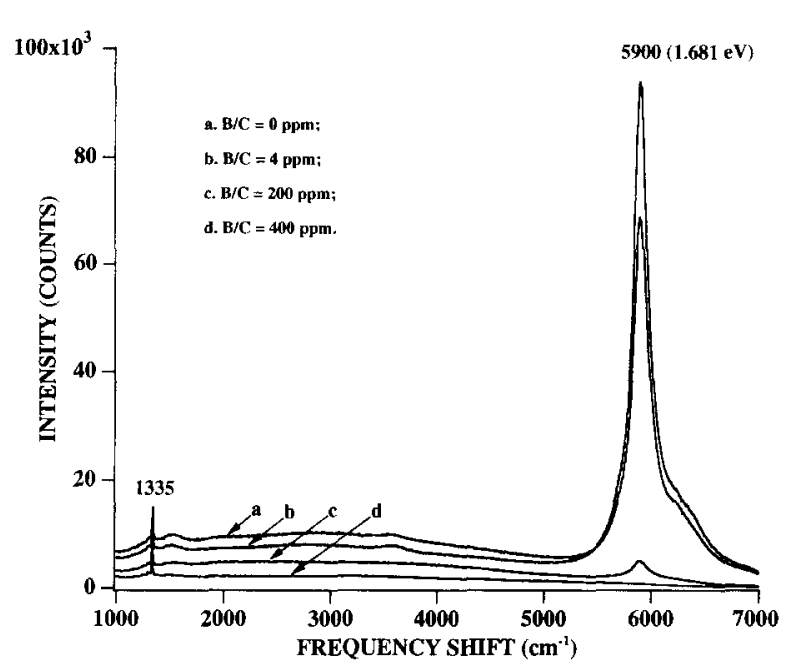

Fig. 5. Photoluminescence spectra of diamond films grown at different $\mathrm{B} / \mathrm{C}$ ratios. The $\mathrm{PL}$ peak at $1.681 \mathrm{eV}(738 \mathrm{~nm})$ decreased and essentially disappeared in the boron-doped film.

indicated that the prominent $\mathrm{PL}$ peak at $1.681 \mathrm{eV}$ $(738 \mathrm{~nm})$ which is common for undoped CVD diamond films on silicon substrates decreased with the addition of boron atoms. This peak essentially disappeared in the heavily doped film (e.g. by B/C ratio at $400 \mathrm{ppm}$ ). The PL feature at $1.681 \mathrm{eV}$ from CVD diamond is very close to the GR 1 line at $1.674 \mathrm{eV}$ common in natural diamond and associated with neutral vacancies [22]. Thus, it has been speculated in the literature that it is related to similar point defects (vacancies) in CVD diamond films as the GR1 center in natural diamond $[14,23,24]$.

\section{Discussions}

\subsection{Nucleation enhancement}

It is obvious that boron doping enhanced the nucleation density and decreased the grain size of diamond films, as shown in Fig. 1. There are similar reports [25-27] on the effects of boron doping on the growth of silicon films. Numerous attempts have been made to explain this behavior. The catalytic influence of adsorbed boron atoms on the growth kinetics (gas phase decomposition and adsorption rates) are considered to be the main reason for such favorable effects on the surface of silicon. Similar ideas on the catalytic effects of boron have also been put forward for the nucleation and growth of CVD diamond films [28] but the detailed mechanisms remain unknown. Most probably, the effect of nucleation enhancement with the boron addition is due to a number of possible mechanisms: (1) The boron atoms catalytically promote the dissociation of carboncontaining molecules and enhance the creation of the correct diamond nucleation species. (2) The positive potential which adsorbed boron acceptors bring to the surface induces electron bombardment from the plasma. The electron bombardment has long been known for its favorable effects of enhancing adatom mobility and chemical reactions on the surface $[23,29]$. (3) Boron reacts with other species (such as hydrogen, nitrogen and silicon, all of which were identified by SIMS). These side reactions disrupt the normal diamond growth and consequently increase the diamond nucleation. (4) Along the same line, since boron was found to enter the diamond lattice at a lower rate than carbon, the boron atoms reduce the growth rate of existing diamond grains on the silicon substrate and thus enhance the nucleation probability of new diamond grains. However, more theoretical modeling and carefully planned experiments are needed to examine these possible mechanisms and reach a conclusion as to the mechanism(s) responsible for the nucleation enhancement by boron doping.

\subsection{Line and planar defect density decrease}

Bernholc et al. [30] have indicated that carbon selfdiffusion and vacancy-assisted impurity diffusion in diamond are Fermi level dependent and can be enhanced by dopants because of the lowering of the activation barrier for charged vacancy diffusion. At the diamond growth temperatures $\left(700-900^{\circ} \mathrm{C}\right)$, such an enhanced diffusion with boron incorporation could lead to a higher surface mobility and improved incorporation of the carbon atoms at the growing surface into their proper lattice positions, thereby minimizing the formation of structural defects in the films. Such phenomena of enhanced surface diffusion leading to minimized structural defects have been well documented in the literature. For example, Ma et al. [31] found better structural quality of diamond films grown at higher substrate temperatures. Based on the results, they indicated that the enhanced surface mobility of carbon atoms, which occurred at higher substrate temperatures, allowed atoms to arrange themselves into their equilibrium surface state prior to overgrowth without the $60^{\circ}$ bond rotation (this rotation creates a $\{111\}$ twin boundary or stacking fault) and thus resulted in improved quality. This is also similar to the reported effect of boron on the densification of $x$-SiC through the sintering process [32]. The presence of boron enhances both the lattice and grain-boundary diffusion rates to the extent that they allow full densification in the $\alpha-\mathrm{SiC}$ which densifies only slightly in the pure state. Thus, one reasonable explanation for the boron-doping effects here involves enhanced surface diffusion during growth, thereby enabling an improvement in the structural quality. The reduced growth rate of diamond films upon boron additions may also play a role in allowing sufficient diffusion and reducing defects.

In addition, recent semi-empirical molecular orbital calculations by Mainwood [33] showed that the total 
energy change upon boron substitution into diamond is minimal. This suggests that the boron substitution into the diamond carbon lattice is a very favorable and stable process. Furthermore, the kinetics of the classical hydroboration reaction in organic chemistry (a reaction involves the addition of borane to carbon-carbon double bonds) [34, 35] suggests that boron is an effective catalyst in breaking the carbon-carbon double bonds and polymerizing the molecules. Therefore any carboncarbon double bonds on the growing diamond surface may be eliminated by the presence of borane species (containing hydrogen and boron atoms) in the plasma. It has also been reported that the bonding probability of boron atoms with $\mathrm{sp}^{2}$ hybridized carbon atoms is negligible at the deposition temperature [36]. As a result, the adsorbed boron atoms are expected to have a similar effect to atomic hydrogen in stabilizing the $\mathrm{sp}^{3}$ hybridization of carbon atoms on the growing surface and ensuring the growth of good quality diamond films, although the number of boron atoms was much smaller than the number of hydrogen-terminated bonds.

\subsection{Point defect density decrease}

The population of point defects (vacancies) was decreased with the introduction of the boron dopant (Fig. 5). For CVD diamond films grown on silicon substrates, these vacancy centers have been speculated to be introduced by the incorporation of silicon atoms during the growth $[14,23,24]$. If this is the case, the boron atoms can occupy either the vacant sites created by the large silicon atoms or the normal diamond lattice adjacent to the vacancy. With these atomic configurations, the optically active defects are effectively passivated or quenched by the boron atoms. Consequently, the extra energy level responsible for the PL feature is altered or eliminated. Similar phenomena have been observed by Dyer and Ferdinando [37] in semiconducting natural diamond. These indicated that all acceptors in $p$-type diamond have to be compensated before absorption or luminescence spectra can be measured. In addition, the increase in carbon self-diffusion discussed in the preceding section may also lower the point defect density by allowing the vacancies to migrate to surfaces during growth. The determination of which of the preceding speculations is dominant remains an interesting topic of investigation.

\section{Summary}

Boron dopants were effectively introduced into diamond films by mixing diborane with hydrocarbon/ hydrogen gases in a microwave plasma enhanced CVD process. The diamond grain sizes were significantly reduced with addition of boron. Furthermore, an increas- ing degree of structural perfection of diamond was achieved by the boron dopant. Graphitic components and planar defects were reduced, and optically active point defects were eliminated. These improvements in quality occurred despite the fact that grain sizes decreased (and thus grain boundary area increased) and $\{111\}$ faceting increased with boron additions (the $\{111\}$ faces are known to contain higher defect density). Further work is needed to study in detail the gas phase and surface chemistry in the boron-containing environment and to clarify the responsible mechanisms for the boron effects.

\section{Acknowledgments}

The authors thank Dr K. Kobashi and his co-workers in the Electronics Research Laboratory, Kobe Steel, Ltd., Japan for preparing the diamond films for this research. They are also grateful to $\mathrm{Dr}$ K. Das for providing standards for SIMS analysis. Dr R. F. Davis is acknowledged for useful technical discussions. This research was supported by the New Energy and Industrial Technology Development Organization (NEDO) with the Ministry of International Trade and Industry (MITI) of Japan. TEM/EELS analysis was supported in part by the Division of Materials Sciences, US Department of Energy through the SHaRE Program under contract DE-AC05-76OR00033 with Oak Ridge Associated Universities.

\section{References}

1 N. Fujimori, T. Imai and A. Doi, Vacuum, 36 (1986) 99.

2 K. Okano, H. Naruki, Y. Akiba, T. Kurosu, M. Iida, Y. Hirose and T. Nakamura, Jpn. J. Appl. Phys., 28 (1989) 1066.

3 K. Kobashi, K. Nishimura, K. Miyata, Y. Kawate, J. T. Glass and B. E. Williams, in A. Feldman and S. Holly (eds.), SPIE Proceedings on Diamond Optics, Vol. 969, San Diego, CA, 1988, pp. 159.

4 J. Mort, D. Kuhman, M. Machonkin, M. Morgan, F. Jansen and K. Okumura, Appl. Phys. Lett., 55 (1989) 1121.

5 N. Fujimori, H. Nakahata and T. Imai, Jpn. J. Appl. Phys., 29 (1990) 824.

6 K. Nishimura, K. Das and J. T. Glass, J. Appl. Phys., 69 (I991) 3142.

7 B. E. Williams and J. T. Glass, J. Mater. Res., 4 (1989) 373.

8 B. E. Williams, H. S. Kong and J. T. Glass, J. Mater. Res., 5 (1990) 801.

9 W. Zhu, A. R. Badzian and R. Messier, J. Mater. Res., 4 (1989) 659.

10 W. Zhu, C. Randall, A. R. Badzian and R. Messier, J. Vac. Sci. Technol. A, 7 (1989) 2315.

11 J. Narayan, J. Mater. Res., 5 (1990) 2414.

12 A. Ono, T. Bada, H. Funamoto and A. Nishikawa, Jpn. J. Appl. Phys., 25 (1986) L808.

13 G. Sh. Gildenblat, S. A. Grot, C. R. Wronski, A. R. Badzian, T. Badzian and R. Messier, Appl. Phys. Lett., 53 (1988) 586. 
14 B. G. Yacobi, A. R. Badzian and T. Badzian, J. Appl. Phys., 69 (1991) 1643 .

15 K. Kobashi, K. Nishimura, Y. Kawate and T. Horiuchi, Phys. Rev. B. 38 (1988) 4067 .

16 R. F. Egerton, Electron Energy Loss Spectroscopy in the Electron Microscope, Plenum, New York, 1986, 333 pp.

17 K. F. Turner, B. R. Stoner, L. Bergman, J. T. Glass and R. J. Nemanich, in R. Messier, J. T. Glass, J. E. Butler and R. Roy (eds.), MRS Proceedings on New Diamond Science and Technology, Materials Research Society, Pittsburgh, PA, 1991, p. 607.

18 M. P. Everson and M. A. Tarmor, J. Vac. Sci. Technol. B, 9 (1991) 1570 .

19 Y. M. LeGrice, R. J. Nemanich, J. T. Glass, Y. H. Lee, R. A. Rudder and R. J. Markuns, in J. T. Glass, R. Messier and N. Fujimori (eds.), MRS Proceedings on Diamond, Silicon Carbide and Related Wide Bandgap Semiconductors, Materials Research Socicty, Pittsburg, PA, Vol. 162, 1990, p. 219.

20 R. E. Clausing, L. Heatherly and K. L. More, Surf. Coat. Technol. 39/40 (1989) 199.

21 J. L. Kaae, P. K. Gantzel, J. Chin and W. P. West, J. Mater. Res, 5 (1990) 1480

22 G. Davies, Chemistry and Physics of Carbon, Marcel Dekker, New York, 1977, p. 1.

23 A. Sawabe and T. Inuzuka, Appl. Phys. Lett., 46 (1985) 146.

24 V. S. Vavilov, A. A. Gippius, A. M. Zaltsev, B. V. Deryagin, B. V. Spitsyn and A. E. Aleksenko, Sov. Phys. Semicond., 14 (1980) 1078.
25 C. A. Chang, J. Electrochem. Soc..: Solid-State Sci. Technol., 123 (1976) 1245.

26 H. J. Kim and C. V. Thompson, Appl. Phys. Lett., 48 (1986) 399.

27 S. Nakayama, I. Kawashima and J. Murota, J. Electrochem. Soc.: Solid-State Sci. Technol., 133 (1986) 1721.

28 D. J. Poferl, N. C. Gardner and J. C. Angus, J. Appl. Phys., 44 (1973) 1428.

29 A. Sawabe and T. Inuzuka, Thin Solid Films, 137 (1986) 89.

30 J. Bernholc, A. Antonelli and T. M. D. Sole, Phys. Rev. Lett., 61 (1988) 2689.

31 G.-H. M. Ma. Y. Hirose, S. Amanuma, M. McClure, J. T. Prater and J. T. Glass, in R. Messier, J. T. Glass, J. E. Butler and R. Roy (eds.) MRS Proceedings on New Diamond Science and Technology, Materials Research Society, Pittsburgh, PA. 1991, p. 587.

32 J. E. Lane. C. H. Carter, Jr. and R. F. Davis, J. Am. Ceram. Soc., 7/ (1988) 281 .

33 A. Mainwood, J. Phvs. C: Solid State Phvs., 12 (1979) 2543.

34 F. A. Carey and R. J. Sundberg, Advanced Organic Chemistry, Part B. Reactions and Synthesis, Plenum, New York and London, 1977 , p. 100 .

35 M. F. Lappert, in E. L. Muetterties (ed.), The Chemistry of Boron and Its Compounds, Wiley, New York, 1967, p. 443.

36 S. Marinkovic, Chemistry and Physics of Carbon, Marcel Dekker, New York, 1984, p. 1.

37 H. B Dyer and P. Ferdinando, Br. J. Appl. Phys., 17 (1966) 419. 